WestVirginiaUniversity
THE RESEARCH REPOSITORY @ WvU

Faculty Scholarship

2013

\title{
On phase diagrams of magnetic reconnection
}

P. A. Cassak

J.F. Drake

Follow this and additional works at: https://researchrepository.wvu.edu/faculty_publications

\section{Digital Commons Citation}

Cassak, P. A. and Drake, J. F., "On phase diagrams of magnetic reconnection" (2013). Faculty Scholarship. 261.

https://researchrepository.wvu.edu/faculty_publications/261

This Article is brought to you for free and open access by The Research Repository @ WVU. It has been accepted for inclusion in Faculty Scholarship by an authorized administrator of The Research Repository@ WVU. For more information, please contact ian.harmon@mail.wvu.edu. 


\title{
On phase diagrams of magnetic reconnection
}

\author{
P. A. Cassak ${ }^{1}$ and J. F. Drake ${ }^{2}$ \\ ${ }^{1}$ Department of Physics, West Virginia University, Morgantown, West Virginia 26506, USA \\ ${ }^{2}$ IREAP, University of Maryland, College Park, Maryland 20742-3511, USA
}

(Received 6 October 2012; accepted 29 November 2012; published online 17 June 2013)

\begin{abstract}
Recently, "phase diagrams" of magnetic reconnection were developed to graphically organize the present knowledge of what type, or phase, of reconnection is dominant in systems with given characteristic plasma parameters. Here, a number of considerations that require caution in using the diagrams are pointed out. First, two known properties of reconnection are omitted from the diagrams: the history dependence of reconnection and the absence of reconnection for small Lundquist number. Second, the phase diagrams mask a number of features. For one, the predicted transition to Hall reconnection should be thought of as an upper bound on the Lundquist number, and it may happen for considerably smaller values. Second, reconnection is never "slow," it is always "fast" in the sense that the normalized reconnection rate is always at least 0.01 . This has important implications for reconnection onset models. Finally, the definition of the relevant Lundquist number is nuanced and may differ greatly from the value based on characteristic scales. These considerations are important for applications of the phase diagrams. This is demonstrated by example for solar flares, where it is argued that it is unlikely that collisional reconnection can occur in the corona. (C) 2013 AIP Publishing LLC. [http://dx.doi.org/10.1063/1.4811120]
\end{abstract}

\section{INTRODUCTION}

During magnetic reconnection, a change in magnetic topology facilitates a conversion of magnetic energy into kinetic and thermal energy. It occurs in solar flares, magnetospheric events, magnetically confined fusion devices, and many astrophysical settings. Despite being known for nearly 60 years, ${ }^{1}$ it remains an unsolved grand challenge problem in plasma physics because it entails dynamics on multiple length and time scales. There have been many recent reviews $^{2-11}$ and books ${ }^{12-14}$ on various aspects of reconnection.

One aspect enjoying renewed interest is the way reconnection is manifested in different parameter regimes and predicting the parameters for which each manifestation, or phase, occurs. The phases in question are the Sweet-Parker phase, the secondary island phase in which collisional reconnection operates with self-consistently generated plasmoids, and the collisionless Hall phase.

The Sweet-Parker phase ${ }^{15,16}$ occurs when collisions break the frozen-in condition. This model has been confirmed in many simulations ${ }^{17,18}$ and laboratory experiments. ${ }^{19-21}$ However, it was realized early on that this phase is much slow to explain observed energy release rates in solar flares when extrapolated to coronal parameters. ${ }^{22}$ One thrust of reconnection research has been to learn what makes reconnection faster than the Sweet-Parker phase for high Lundquist number plasmas.

The Petschek model allowed faster reconnection due to energy being converted at an open outflow region outside a tiny dissipation region, ${ }^{23}$ but simulations showed it does not occur with a uniform resistivity. ${ }^{17,18}$ It can occur due to a localized resistivity, ${ }^{24-26}$ but since the fundamental physics of what localizes the resistivity is not well understood, it will not be further considered here. However, spawning from numerical work on tokamaks, it was shown that collisionless reconnection is faster than Sweet-Parker reconnection. ${ }^{27}$ The Hall term is sufficient to develop the Petschek open outflow region and causes reconnection to be fast. ${ }^{28}$ The reconnection rate in the Hall phase is independent of system size with a normalized value of $E \simeq 0.1,{ }^{29}$ which is comparable to observed energy release rates in flares and substorms. ${ }^{30}$ There is now copious evidence from magnetospheric observations $^{31-38}$ and laboratory experiments ${ }^{39-42}$ that Hall reconnection occurs.

The landscape recently changed with a better appreciation for the secondary island phase. It was long known that when the Lundquist number $S$ exceeds $10^{4}$, Sweet-Parker reconnection fundamentally changes through the selfconsistent appearance of plasmoids. ${ }^{17}$ Recent work showed the growth of plasmoids is rapid. ${ }^{43,44}$ Plasmoids make reconnection rates faster, ${ }^{45,46}$ and numerical work thus far gives normalized reconnection rates very close to $0.01 .^{44,47-49}$ This reconnection rate greatly exceeds the Sweet-Parker prediction for large Lundquist numbers. It is critical to understand how secondary islands affect reconnection.

To address this issue, the present knowledge of the phases of reconnection as a function of system parameters has been gathered in "phase diagrams." have proven useful to predict which phase dominates for particular systems. However, there are a number of cautions for applying them to real systems; we present two examples of physics omitted from the diagrams and a number of potential pitfalls that should be remembered when using the diagrams.

The construction and caveats of existing phase diagrams are reviewed in Sec. II. A modified phase diagram is presented in Sec. III; two omissions from existing diagrams are highlighted. Section IV contains a number of cautions when interpreting the phase diagrams. Section V applies the diagrams to solar flares, showing that collisional reconnection is 
unlikely to occur in the solar corona and that the onset of solar flares is unlikely to be explained solely by the microphysics of two-dimensional reconnection. Open questions are summarized in Sec. VI. To keep the treatment manageable, aspects of reconnection outside of phase diagrams are not treated here.

\section{THE PHASE DIAGRAMS}

An example of a phase diagram of magnetic reconnection is shown in Fig. 1. This is from Ref. 51, but the same qualitative and quantitative picture was shown contemporaneously in Refs. 5 and 50. The diagrams are parametrized as a function of two system parameters: the Lundquist number $S=4 \pi c_{A} L / \eta c^{2}$ on the vertical axis and the ratio of the system size $L$ to the kinetic length scale $d_{k}$, which is called $\lambda=$ $L / d_{k}$ on the horizontal axis in Fig. 1. Here, $c_{A}$ is the Alfvén speed and $\eta$ is the classical Spitzer resistivity (in cgs units). The kinetic scale $d_{k}$ is the ion inertial scale $d_{i}=c / \omega_{p i}$ when the reconnection is anti-parallel (there is no out-of-plane "guide" magnetic field) ${ }^{52-54}$ and the ion Larmor radius $\rho_{s}=$ $c_{s} / \Omega_{c i}$ when the reconnection is component (there is a strong guide field). ${ }^{53,55-58}$ Characteristic parameters for many settings have been plotted on the diagram in Fig. $1 .^{51}$

In Fig. 1, the phases discussed in Sec. I are represented. The Sweet-Parker phase dominates for small $S$ (below the green line, referred to as "Single X-line collisional"). For most parameter regimes, an increase in $S$ at fixed $\lambda$ leads to the self-consistent generation of secondary islands or plasmoids (between the green and blue lines, which is referred to as "Multiple X-line collisional"). Continuing to higher $S$, the secondary islands lead to current sheets at the kinetic scale, which induces a transition to the Hall phase (between the blue and black lines, which is referred to as "Multiple X-line hybrid"; this terminology will be discussed later). Finally at high $S$, reconnection is "collisionless" and the Hall phase proceeds (above the black line). The orange line gives an approximate distinction of the number of $\mathrm{X}$-lines expected in the Hall phase; we do not treat this transition further.

The transitions from phase to phase are drawn as abrupt, which is based on previous simulation results. ${ }^{50,54,58,59}$ The lines are calculated as follows. The empirically determined condition for the transition from the Sweet-Parker to secondary island phase is ${ }^{17}$

$$
S>S_{\text {crit }} \simeq 10^{4}
$$

This is drawn as the green line.

For the transition from the secondary island to the Hall phase, it occurs when the thickness of the current layer is smaller than $d_{k}$. It was argued that the splintering of a SweetParker current sheet by secondary islands leads to SweetParker-like reconnection in the fragmented current sheets, ${ }^{47,60}$ so the thickness of the current sheet on average is the standard Sweet-Parker thickness $\delta_{S P}$ divided by the square root of the number of plasmoids $N{ }^{48}$ Simulations thus far suggest that the number of islands scales as $N \sim S / S_{\text {crit }},{ }^{44,48,49}$ so the transition to the Hall phase occurs when this thickness equals the kinetic scale $d_{k}$

$$
\frac{\delta_{S P}}{\sqrt{N}} \simeq d_{k} .
$$

Using $\delta_{S P} \sim L / \sqrt{S},{ }^{16}$ this can be written as $S \simeq 100 \lambda$, which is plotted as the blue line. Note, there is an ambiguity in the literature about the definition of $L$; some call this the characteristic system size, while others call it the half-length of the Sweet-Parker layer which is likely to be half or a quarter of the characteristic system size. We ignore this ambiguity.

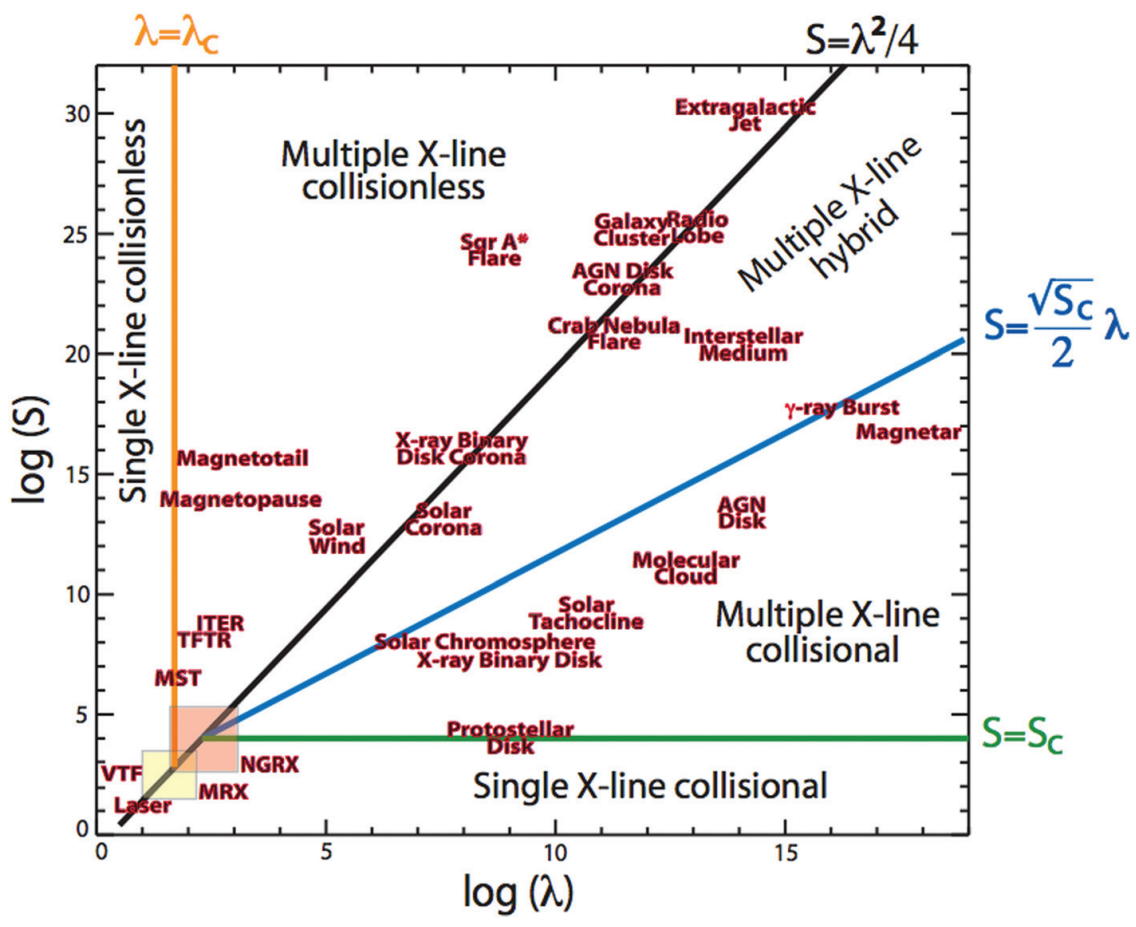

FIG. 1. Phase diagram for reconnection. Reprinted with permission from Phys. Plasmas 18, 111207 (2011). Copyright 2011 American Institute of Physics. See also similar diagrams in Refs. 5 and 50. 
Finally, collisional reconnection does not occur if the Sweet-Parker thickness is smaller than the kinetic scale $d_{k}$; the cutoff occurs when

$$
\delta_{S P} \simeq d_{k}
$$

In such a regime, the Hall phase proceeds without collisional plasmoids ever occurring. This can be written as $S \simeq \lambda^{2}$, which is plotted as the black line.

It should be noted that a number of assumptions are used in the creation of the phase diagrams, which have been noted previously. First, the theory is based on twodimensional (2D) steady-state reconnection theory and simulations; three-dimensional (3D) effects are not included. ${ }^{5,51}$ Asymmetries and shear flow effects are not included. ${ }^{50,51}$ Reconnection sites are isolated; no line tying or other external effects that interfere with reconnection setting up naturally are included. ${ }^{51}$ Reconnection is assumed to be laminar ${ }^{5}$ in the sense that incoming magnetic field lines have not been disturbed (though the self-consistent production of secondary islands may feed back and disturb the upstream field lines ${ }^{46}$ ). Thus, results on so-called turbulent reconnection ${ }^{61-68}$ are not captured by the diagram. Also, the figure extrapolates what has heretofore been determined from simulations; 5,50 computational limitations have prevented studies above $S \simeq 10^{6}$ and $\lambda \simeq 10^{3}-10^{4}$.

\section{OMISSIONS FROM PHASE DIAGRAMS}

Even within the context of 2D laminar reconnection, existing phase diagrams do not include two physical aspects of reconnection. Before considering applications, it is worthwhile to ensure the diagrams capture all known results on reconnection. A modified phase diagram taking these additional considerations into account is shown in Fig. 2; the new features are discussed in the following subsections.

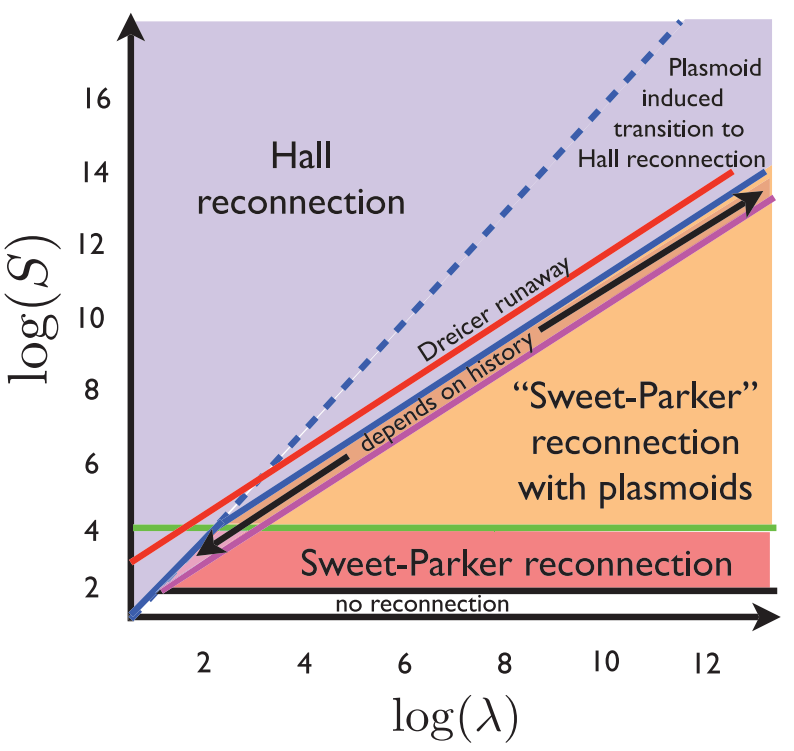

FIG. 2. Modified phase diagram of 2D reconnection including the history dependence of reconnection and no reconnection for small $S$.

\section{A. No reconnection for small $S$}

Existing phase diagrams suggest the Sweet-Parker phase occurs for arbitrarily small Lundquist number. This is unlikely the case-as the collisionality increases, at some point magnetic diffusion dissipates energy fast enough that reconnection does not occur. It was suggested on theoretical grounds that this cutoff is near $S \sim 100$. $^{69}$ The precise cutoff has not been confirmed numerically, but it is unlikely to be of import for space or fusion applications. Nonetheless, this facet of reconnection should be included in the diagram; this is represented by the white band labeled "no reconnection" at low $S$ in Fig. 2. In this plot, the cutoff has been drawn as the black line independent of $\lambda$ at $S=100$.

\section{B. History dependence of reconnection}

By construction, the phase diagram is intended to prescribe the phase of reconnection for a given set of system parameters. This assumes that a given set of parameters leads to a unique phase of reconnection. However, this is not the case $;{ }^{54}$ there are parameter regimes for which reconnection is bistable, i.e., the reconnection phase is history dependent. ${ }^{50,54,58,59,70}$ This occurs because the physical condition necessary to cause a transition from a collisional phase to the Hall phase is different from the condition to transition back. ${ }^{54}$ The implication for the phase diagram is that it is not possible to say that the reconnection phase is determined fully by the two parameters $S$ and $\lambda$.

Existing diagrams give transitions from the SweetParker and secondary island phases to the Hall phase, but not the back-transition from the Hall-phase to the collisional phases. This transition was predicted previously, ${ }^{54}$ but the analysis did not include the effect of secondary islands. Since secondary islands arise when $S>S_{\text {crit }}$, the previous analysis should remain valid for $\lambda<100$ since plasmoids do not arise in such systems.

For larger systems with $\lambda>100$, one expects the backtransition from the Hall phase to the secondary island phase is the same condition as for the back-transition to the SweetParker phase ${ }^{50}$ since the effect causing the transition is for resistivity to dominate at the electron scale. ${ }^{54,58}$ This occurs when

$$
\frac{\eta c^{2}}{4 \pi \delta^{2}} \sim \frac{v_{i n, e}}{\delta}
$$

where $\delta$ is the thickness of the electron dissipation region and $v_{i n, e}$ is the inflow speed at the electron layer. Using $\delta \sim$ $d_{e}$ for collisionless reconnection ${ }^{71}$ and $v_{\text {in }, e} B_{\text {up }, e} \sim v_{\text {in }, i} B_{\text {up }, i}$ from the constancy of the reconnection electric field in the steady-state (where $B_{u p, i}$ and $B_{u p, e}$ are the magnetic field strengths upstream of the ion and electron layers, respectively) allows us to write Eq. (4) as

$$
S \sim \frac{c_{A} B_{u p, e} d_{i}}{v_{\text {in }, i} B_{\text {up }, i} d_{e}} \lambda
$$

Since $v_{i n, i} \sim c_{A} / 10$ (Refs. 28, 29, 52, and 72-74) during the Hall phase 


$$
S \sim 10 \frac{B_{u p, e} d_{i}}{B_{u p, i} d_{e}} \lambda
$$

The fraction multiplying $\lambda$ is a function of electron physics which is not expected to change much across parameters but should be evaluated for any given system. To be definite for the purposes of plotting on the phase diagram, experience from simulations ${ }^{54,75}$ suggests that a reasonable estimate for the fraction is approximately 2 , which gives

$$
S \sim 20 \lambda
$$

for the back-transition from the Hall to collisional reconnection phases. A number of numerical studies do not employ electron inertia; a similar analysis for the back transition condition would have to be carried out for such systems.

The dynamical behavior of reconnection is conveniently plotted in a bifurcation diagram displaying the normalized reconnection rate $E$ as a function of $\eta c^{2} / 4 \pi c_{A} L=1 / S$. Figure 3(a) shows a bifurcation diagram for $\lambda<100$. For the Sweet-Parker branch, consider a system starting with $S$ very small and increasing. Until $S$ exceeds 100 or so, no reconnection occurs. After this, Sweet-Parker reconnection occurs with its characteristic $1 / S^{1 / 2}$ reconnection rate, drawn as the solid line. The system continues in this phase until the thickness of the layer $\delta_{S P}$ crosses kinetic scales, given by Eq. (3) as $S=\lambda^{2}$ and marked by the left-most tick in panel (a). Below this value, the Sweet-Parker phase cannot occur, and the system must be in the Hall phase. Conversely, a system with $S$ decreasing from high values stays in the Hall phase until Eq. (7) is satisfied, where it transitions back to the Sweet-Parker phase. Between an $S$ of $20 \lambda$ and $\lambda^{2}$,
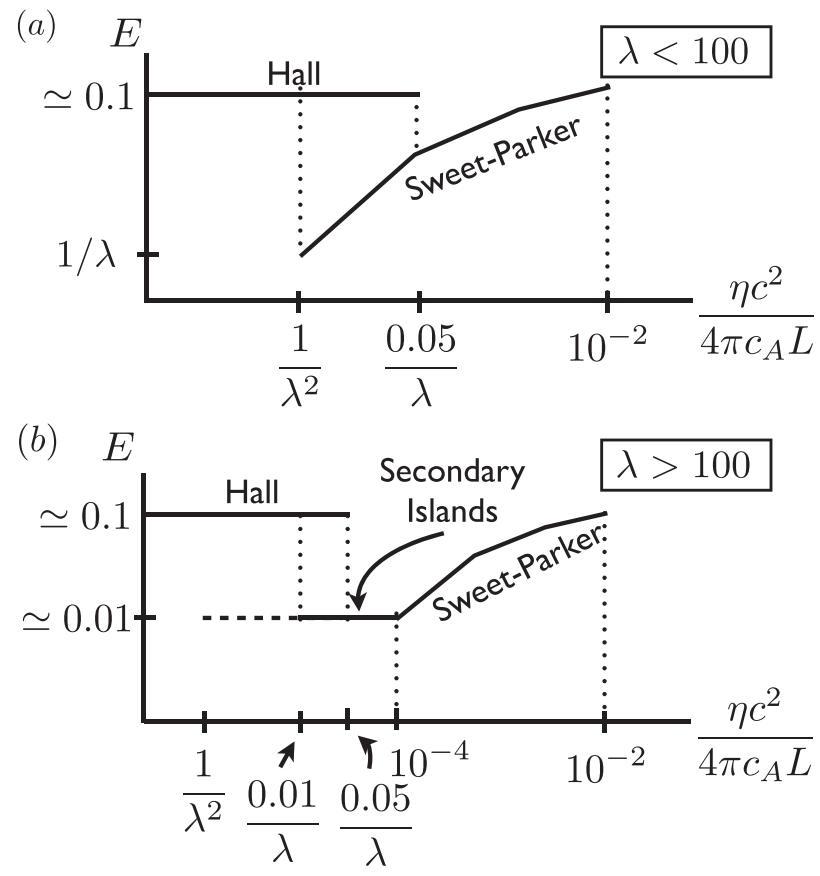

FIG. 3. Expected bifurcation diagram of 2D reconnection for (a) $\lambda<100$ (sketched for $\lambda \simeq 50$ ) adapted from Refs. 54 and 70 and (b) $\lambda>100$ (sketched for $\lambda \simeq 1,000$ ). reconnection is bistable. This has been verified numerically $^{50,54,58,59}$ and was described as a saddle-node bifurcation. $^{70}$

For $\lambda>100$ where secondary islands occur, the bifurcation diagram is plotted in Fig. 3(b). For the Sweet-Parker branch, there is no change until $S$ reaches $S_{\text {crit }} \simeq 10^{4}$. At this point, the secondary island phase begins with a reconnection rate of 0.01 . This continues until the current sheets reach kinetic scales, which is given in Eq. (2) as $S \sim 100 \lambda$. Above this $S$ value, the system can only be in the Hall phase. The horizontal dashed line denotes that plasmoids transiently appear, but a transition to the Hall phase quickly occurs. For the Hall branch, the dynamics is the same as for $\lambda<100$; the Hall phase cannot occur below $S=20 \lambda$. A simple calculation reveals that the thickness of the layer after this transition exceeds the kinetic scale, so the system is in the plasmoid phase. As $S$ is decreased below $10^{4}$, the system transitions back to the Sweet-Parker phase. From the bifurcation diagram, we see that there is bistability for $S$ between $20 \lambda$ and $100 \lambda$. Note that a much smaller range of parameter space is bistable when $\lambda<100$. As will be discussed in Sec. IV B, the transition to the Hall phase at $S \sim 100 \lambda$ is actually an upper bound on the transition, so the region with bistability could be smaller than predicted or even not exist entirely for large $\lambda$.

The transition from Hall to collisional reconnection from Eq. (7) is plotted on the phase diagram as the purple line in Fig. 2. The region below the blue line and above the purple line is where reconnection is history dependent, as is labeled on the diagram. The placement of the blue line will be discussed further in Sec. IV B. We note in passing that Refs. 5 and 51 also marked the parameters for which the reconnection electric field exceeds the Dreicer field, precluding any type of collisional reconnection from occurring (with a classical resistivity). This is plotted as the red line in Fig. 2.

In conclusion, the phase diagram is not a plane that uniquely identifies a particular phase with system parameters. Rather, the diagram has a double valued cusp catastrophe-type structure, as sketched in Fig. 4, which allows there to be either of two stable solutions for given

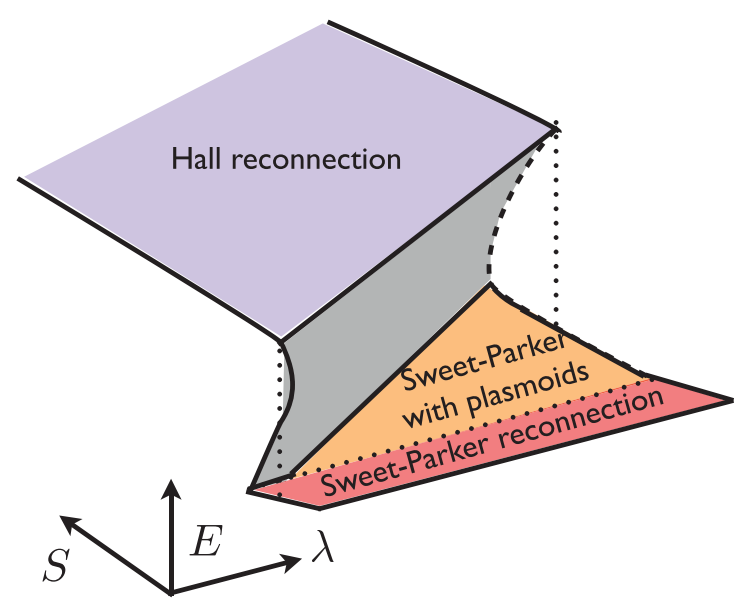

FIG. 4. Phase diagram of reconnection including history dependence of reconnection. The vertical axis denotes reconnection rate $E$. 
parameters. The sketches in Fig. 3 can be interpreted as slices of the cusp catastrophe diagram at fixed $\lambda$ (a) smaller or (b) larger than 100. It is expected that there is an unstable branch between the two stable ones; this has been found for $\lambda<100$ (Ref. 76) but not for $\lambda>100$.

\section{INTERPRETATION CAUTIONS}

The phase diagram is invaluable as a way to organize the known results on $2 \mathrm{D}$ reconnection, but there are a few places where it could engender inappropriate interpretations. In this section, we discuss a number of issues.

\section{A. The "plasmoid induced transition to Hall reconnection" is not a separate phase}

In the phase diagrams to date, the plasmoid induced transition to Hall reconnection is given its own region in the phase diagram; this is the part labelled "Multiple $\mathrm{X}$-line hybrid" between the blue and black lines in Fig. 1. We argue that this regime is not genuinely a separate phase of reconnection. Rather, in such a system, if it is begun with a current sheet wider than kinetic scales, the initial burst of reconnection will arise from collisional reconnection with secondary islands. However, these secondary islands immediately take the current sheet thickness below kinetic scales. Hall reconnection begins, and the system remains in the Hall phase for the remainder of the evolution. Therefore, except for a very short lived transient phase at onset, the phase of reconnection in this wedge is essentially indistinguishable from "pure" Hall reconnection (above the black line in Fig. 1). To emphasize this point, the wedge in question has been colored the same as the Hall phase in Fig. 2. The title "Multiple X-line hybrid" is not appropriate for this wedge, and it should be considered a part of the Hall phase.

\section{B. The secondary island to Hall transition is an upper bound on $S$}

The blue line in Figs. 1 and 2 denotes the transition from the secondary island phase to the Hall phase when secondary islands introduce length scales below kinetic scales, as predicted in Eq. (2). However, this prediction should be thought of as an upper bound on the transition; it may be much lower on the diagram as discussed in Ref. 50.

The reason is that the theory used to develop Eq. (2) is based on $N$ secondary islands breaking the current sheet into pieces of length $L / N$. This is true on average, ${ }^{48,49}$ but significant deviations from the mean occur. ${ }^{50}$ The result of these deviations is that some current sheet segments are shorter and some are longer, and the shorter ones will be thinner and arrive at kinetic scales before longer segments do. Once even one reconnection site transitions to the Hall phase, the Hall phase quickly dominates the dynamics. ${ }^{47,77}$ After the transition to the Hall phase, it is likely to stay in that phase (though it has been suggested that it can flip back and forth with the secondary island phase ${ }^{50}$ ).

As a result, the blue line in the diagram should be treated as an upper bound, and it could actually be significantly lower. ${ }^{50}$ Depending on how low the transition goes, the region of bistability could completely disappear. However, this will only be the case for large $\lambda$, and bistability continues to persist for smaller $\lambda$. This process has not been studied sufficiently well to make a reliable prediction of where the line should be, but a preliminary numerical study has confirmed that the back-transition occurs earlier than expected from Eq. (7). ${ }^{78}$ This will be an important aspect for future study.

\section{2D reconnection is always fast!}

The failure of the Sweet-Parker model to explain observed energy release rates in solar flares and fusion devices led to the search for faster forms of reconnection. SweetParker reconnection has become synonymous with "slow" reconnection. "Fast" reconnection has come to mean anything with a reconnection rate of 0.01 or 0.1 when extrapolated to realistic parameters, and "slow" reconnection is anything that remains much slower.

The planar phase diagrams (Figs. 1 and 2) encourage this interpretation by not showing the reconnection rate for these phases. Note, however, that the Sweet-Parker phase is only "slow" when the Sweet-Parker model is extrapolated to high $S$ conditions.

The situation is seen more clearly in the bifurcation diagrams in Fig. 3. Sweet-Parker reconnection only ever occurs for $S<10^{4}$. Therefore, the reconnection rate of SweetParker reconnection $E \simeq 1 / S^{1 / 2}$ always exceeds 0.01 . This is a "fast" reconnection rate! This implies that within the known results of $2 \mathrm{D}$ reconnection, there is no such thing as "slow reconnection" if defined by the normalized reconnection rate. Implications for understanding reconnection in the corona are discussed in Sec. V.

\section{The definition of $S$ is nuanced}

The phase diagram has been used to assess what phase of reconnection occurs for given systems by finding characteristic values of the magnetic field and density (to estimate the Alfvén speed and kinetic length scales), temperature (to estimate the resistivity), and system sizes to see where these conditions lie in the phase diagram. It is well known that characteristic values can be useful to get a big picture idea but can be misleading for specific applications. Here we explicitly give examples of this as well as some more subtle complications that can arise.

The Lundquist number $S=4 \pi c_{A} L / \eta c^{2}$ of import to reconnection requires information from different locations. The resistivity is a strong function of temperature ${ }^{79}$ and depends on density; these values should be the characteristic values at the current sheet locally where the reconnection occurs. These values may or may not be the same as largescale characteristic values. On the other hand, the length scale $L$ is a characteristic global scale which is a function of external effects, such as footpoint motion in coronal contexts, ${ }^{80-82}$ which may be difficult to predict for given systems and even evolve in time.

For the magnetic field strength, it has been shown in a number of numerical studies that it should be measured immediately upstream of the current sheet ${ }^{83-86}$ because this is 
the field strength that determines the rate of reconnection. This value may be different than a global characteristic scale; when this is the case, the reconnection is said to be embedded. ${ }^{83}$ Since the upstream field can be very different and the reconnection rate depends on a positive power of the magnetic field strength $\left(B^{2}\right.$ for the Hall and secondary island phases, $B^{3 / 2}$ for the Sweet-Parker phase), this is potentially very important in determining the expected rate of reconnection for a given system.

This raises an important question about the evolution of reconnection. One might expect the Lundquist number for the reconnection site to start small and increase due to external motions increasing the magnetic field and/or system size. Thus, it is unclear whether the high $S$, high $\lambda$ portion of the phase diagram is ever relevant. ${ }^{87}$ It is very unlikely that, for example, a current sheet with $S \sim 10^{8}$ exists when $\lambda \sim 10^{10}$; it is more likely that a current sheet forms and undergoes reconnection at a lower $S$ (perhaps when $S \sim S_{\text {crit }}$ ), and subsequent smaller layers become unstable in a hierarchical production of islands. ${ }^{5,47,51,60}$ While islands of various sizes have been reported, ${ }^{49}$ hierarchical island formation in the picture of Ref. 60 has not been observed. A reasonable theory of reconnection in such systems has been presented, ${ }^{51}$ but numerical validation is not yet complete.

\section{IMPLICATIONS FOR SOLAR FLARES}

As an example of how to use the phase diagrams, consider solar flares. In Fig. 1, the solar corona lies significantly (4-5 orders of magnitude) above the blue line, which puts it firmly into the Hall phase (see also Fig. 2). Therefore, the diagram suggests that reconnection only occurs in the Hall phase in the corona, and it is unlikely that collisional reconnection can occur other than transiently.

Interestingly, plasmoids have been observed in coronal current sheets. ${ }^{88,89}$ Similarly, supra-arcade downflows ${ }^{90,91}$ are downflowing plasma voids from the reconnection site above flare loops and have been suggested to be related to secondary islands. It was shown that they have a distribution of sizes similar to predictions for collisional secondary islands. ${ }^{92}$ However, the scales at which the plasmoids are created are far below the scales that can be resolved in observations, so it is not known whether these plasmoids are a result of collisional or collisionless reconnection. Plasmoids also occur in the Hall phase, ${ }^{.93-95}$ it is unknown whether collisional and collisionless plasmoids are distributed similarly. ${ }^{96,97}$ It is likely they do, so it is not clear that observed plasmoids are evidence of collisional reconnection.

Another important aspect is the onset problem, which addresses why reconnection in flares begins abruptly. An important part of this is understanding why reconnection does not occur while enormous amounts of magnetic energy are being stored. Based on what was known about the transition from the Sweet-Parker to Hall phase, it was suggested that this transition could give the abrupt onset and explain why not much reconnection occurs before a flare. ${ }^{2,54,84}$ However, as discussed in Sec. IV C, reconnection is always fast including during collisional reconnection, so it is not clear how a slow-to-fast transition would work.
One might consider whether embedded effects, as discussed in Sec. IV D could solve this problem. If the upstream magnetic field is small, the normalized reconnection rate could be fast, but the absolute reconnection rate (scaled by $c_{A} B / c$ ) could be slow. There are two problems with this scenario. One is that this evolution is continuous rather than abrupt, so it would have to be carefully shown that the evolution leads to a rapidly changing absolute reconnection rate. The second is that the time scale for a substantial magnetic field to convect into the reconnection site is far smaller than the energy accumulation times, ${ }^{85}$ so fast reconnection (via plasmoids or the Hall effect) would occur too rapidly to allow a significant amount of energy to accumulate.

The conclusion at this point is that the phase diagrams have helped suggest that it is not possible to explain reconnection onset in flares purely from the point of view of $2 \mathrm{D}$ reconnection physics. It seems likely that effects not incorporated into the diagram are necessary. Examples include linetying $^{98,99}$ and effects of the global change in the global magnetic field structure. ${ }^{82,100}$

\section{DISCUSSION}

The introduction of phase diagrams ${ }^{5,50,51}$ into $2 \mathrm{D}$ magnetic reconnection research has provided a simple, visual way to determine which phase of reconnection (SweetParker, collisional reconnection with secondary islands, or Hall) dominates for a given set of system parameters. The only parameters that need to be prescribed are the Lundquist number $S$ and the ratio of the global length scale to the kinetic scale, which has been called $\lambda$.

In this paper, we have discussed a number of aspects of the diagrams that require caution. First, two omissions from the phase diagrams have been noted: reconnection does not occur for small $S$ and reconnection is history dependent. Second, a number of aspects of the diagrams that are prone to misinterpretation have been discussed: plasmoid induced transitions to Hall reconnection should not be considered a distinct phase because the plasmoids occur at early times and are transient, the condition given for the transition from the secondary island phase to the Hall phase is an upper bound rather than precisely where it is drawn in the diagrams so that the transition to the Hall phase may occur for vastly different parameters than the diagrams suggest, the normalized reconnection rate for all phases of reconnection always exceeds 0.01 and is therefore quite fast, and the relevant parameters controlling reconnection are nuanced and may differ from global characteristic scales.

These potential issues are relevant for applications, particularly for solar flares. The results suggest that collisional reconnection is unlikely to occur in the corona for any appreciable time, and it is impossible to explain flare onset within the confines of 2D reconnection theory. Global and 3D effects not incorporated in the phase diagrams likely need to be taken into account to develop a full understanding.

Even within the confines of a phase diagram for $2 \mathrm{D}$ reconnection, many aspects remain to be fully understood. Since the boundary marking the onset of the Hall phase due to secondary islands [the blue line in Figs. 1 and 2] is an 
upper bound, it is extremely important to develop a better understanding of when this transition occurs and how it depends on system parameters. Another very important aspect is verifying the diagram for larger values of $S$ and $\lambda$ than have been able to be accomplished thus far and, more importantly, to determine whether the high $S$, high $\lambda$ regime is ever relevant or whether the evolution through local parameters effectively keeps the system at smaller values.

\section{ACKNOWLEDGMENTS}

P.A.C. gratefully acknowledges support by NSF grant (No. AGS-0953463) and NASA grant (No. NNX10AN08A). This manuscript has been prepared as part of the 2012 USJapan Workshop on Magnetic Reconnection in Princeton, New Jersey in May, 2012. The authors acknowledge many fruitful conversations at this meeting, especially those with M. Swisdak and H. Ji, and thank H. Karimabadi for pointing out a minor error in Fig. 4.

${ }^{1}$ J. W. Dungey, Philos. Mag. 44, 725 (1953).

${ }^{2}$ A. Bhattacharjee, Annu. Rev. Astron. Astrophys. 42, 365 (2004).

${ }^{3}$ E. G. Zweibel and M. Yamada, Annu. Rev. Astron. Astrophys. 47, 291 (2009).

${ }^{4}$ M. Yamada, R. Kulsrud, and H. Ji, Rev. Mod. Phys. 82, 603 (2010).

${ }^{5}$ W. Daughton and V. Roytershteyn, Space Sci. Rev. 172, 271 (2012).

${ }^{6}$ P. A. Cassak and M. A. Shay, Space Sci. Rev. 172, 283 (2012).

${ }^{7}$ M. Hesse, T. Neukirch, K. Schindler, M. Kuznetsova, and S. Zenitani, Space Sci. Rev. 160, 3 (2011).

${ }^{8}$ D. I. Pontin, Adv. Space Res. 47, 1508 (2011).

${ }^{9}$ F. S. Mozer and P. L. Pritchett, Space Sci. Rev. 158, 119 (2011).

${ }^{10}$ D. A. Uzdensky, Space Sci. Rev. 160, 45 (2011).

${ }^{11}$ M. Yamada, Space Sci. Rev. 160, 25 (2011).

${ }^{12}$ D. Biskamp, Magnetic Reconnection in Plasmas (Cambridge University Press, Cambridge, UK, 2000).

${ }^{13}$ E. Priest and T. Forbes, Magnetic Reconnection (Cambridge University Press, 2000).

${ }^{14}$ Reconnection of Magnetic Fields, edited by J. Birn and E. Priest (Cambridge University Press, 2007).

${ }^{15}$ P. A. Sweet, in Electromagnetic Phenomena in Cosmical Physics, edited by B. Lehnert (Cambridge University Press, New York, 1958), p. 123.

${ }^{16}$ E. N. Parker, J. Geophys. Res. 62, 509, doi:10.1029/JZ062i004p00509 (1957).

${ }^{17}$ D. Biskamp, Phys. Fluids 29, 1520 (1986).

${ }^{18}$ D. A. Uzdensky and R. M. Kulsrud, Phys. Plasmas 7, 4018 (2000).

${ }^{19}$ H. Ji, M. Yamada, S. Hsu, and R. Kulsrud, Phys. Rev. Lett. 80, 3256 (1998).

${ }^{20}$ F. Trintchouk, M. Yamada, H. Ji, R. M. Kulsrud, and T. A. Carter, Phys. Plasmas 10, 319 (2003)

${ }^{21}$ I. Furno, T. P. Intrator, E. W. Hemsing, S. C. Hsu, S. Abbate, P. Ricci, and G. Lapenta, Phys. Plasmas 12, 055702 (2005).

${ }^{22}$ E. N. Parker, Ap. J. 8, 177 (1963).

${ }^{23}$ H. E. Petschek, in AAS/NASA Symposium on the Physics of Solar Flares, edited by W. N. Ness (NASA, Washington, DC, 1964), p. 425.

${ }^{24}$ M. Ugai and T. Tsuda, J. Plasma Phys. 17, 337 (1977).

${ }^{25}$ T. Sato and T. Hayashi, Phys. Fluids 22, 1189 (1979).

${ }^{26}$ R. M. Kulsrud, Earth, Planets Space 53, 417 (2001).

${ }^{27}$ A. Y. Aydemir, Phys. Fluids B 4, 3469 (1992).

${ }^{28}$ J. Birn, J. F. Drake, M. A. Shay, B. N. Rogers, R. E. Denton, M. Hesse, M. Kuznetsova, Z. W. Ma, A. Bhattacharjee, A. Otto et al., J. Geophys. Res. 106, 3715, doi:10.1029/1999JA900449 (2001).

${ }^{29}$ M. A. Shay, J. F. Drake, B. N. Rogers, and R. E. Denton, Geophys. Res. Lett. 26, 2163, doi:10.1029/1999GL900481 (1999).

${ }^{30}$ E. N. Parker, Ap. J. 180, 247 (1973).

${ }^{31}$ T. Nagai, I. Shinohara, M. Fujimoto, M. Hoshino, Y. Saito, S. Machida, and T. Mukai, J. Geophys. Res. 106, 25929, doi:10.1029/2001JA900038 (2001).
${ }^{32}$ M. Øieroset, T. D. Phan, M. Fujimoto, R. P. Lin, and R. P. Lepping, Nature (London) 412, 414 (2001).

${ }^{33}$ J. D. Scudder, F. S. Mozer, N. C. Maynard, P. A. Puhl-quinn, Z. W. Ma, and C. T. Russell, J. Geophys. Res. 107, 1294, doi:10.1029/2001JA000126 (2002).

${ }^{34}$ F. Mozer, S. D. Bale, and T. D. Phan, Phys. Rev. Lett. 89, 015002 (2002).

${ }^{35}$ A. Runov, R. Nakamura, W. Baumjohann, R. A. Treumann, T. L. Zhang, M. Volwerk, Z. Vörös, A. Balogh, K.-H. Glabmeier, B. Klecker et al., Geophys. Res. Lett. 30, 1579, doi:10.1029/2002GL016730 (2003).

${ }^{36}$ A. L. Borg, M. Øieroset, T. D. Phan, F. S. Mozer, A. Pedersen, C. Mouikis, J. P. McFadden, C. Twitty, A. Balogh, and H. Rème, Geophys. Res. Lett. 32, L19105, doi:10.1029/2005GL023794 (2005).

${ }^{37}$ T. D. Phan, J. F. Drake, M. A. Shay, F. S. Mozer, and J. P. Eastwood, Phys. Rev. Lett. 99, 255002 (2007).

${ }^{38}$ J. P. Eastwood, T. D. Phan, M. Øieroset, and M. A. Shay, J. Geophys. Res. 115, A08215, doi:10.1029/2009JA014962 (2010).

${ }^{39}$ Y. Ren, M. Yamada, S. Gerhardt, H. Ji, R. Kulsrud, and A. Kuritsyn, Phys. Rev. Lett. 95, 055003 (2005).

${ }^{40}$ C. D. Cothran, M. Landreman, M. R. Brown, and W. H. Matthaeus, Geophys. Res. Lett. 32, L03105, doi:10.1029/2004GL021245 (2005).

${ }^{41}$ M. Yamada, Y. Ren, H. Ji, J. Breslau, S. Gerhardt, R. Kulsrud, and A. Kuritsyn, Phys. Plasmas 13, 052119 (2006).

${ }^{42}$ A. G. Frank, S. Y. Bogdanov, G. V. Dreiden, V. S. Markov, and G. V. Ostrovskaya, Phys. Lett. A 348, 318 (2006).

${ }^{43}$ N. F. Loureiro, A. A. Schekochihin, and S. C. Cowley, Phys. Plasmas 14, 100703 (2007).

${ }^{44}$ A. Bhattacharjee, Y.-M. Huang, H. Yang, and B. Rogers, Phys. Plasmas 16, 112102 (2009).

${ }^{45}$ B. Kliem, in Proceedings of CESRA Workshop, edited by A. O. Benz and A. Krueger, 1995, Vol. 444, p. 93.

${ }^{46}$ G. Lapenta, Phys. Rev. Lett. 100, 235001 (2008).

${ }^{47}$ W. Daughton, V. Roytershteyn, B. J. Albright, H. Karimabadi, L. Yin, and K. J. Bowers, Phys. Rev. Lett. 103, 065004 (2009).

${ }^{48}$ P. A. Cassak, M. A. Shay, and J. F. Drake, Phys. Plasmas 16, 120702 (2009).

${ }^{49}$ Y.-M. Huang and A. Bhattacharjee, Phys. Plasmas 17, 062104 (2010).

${ }^{50}$ Y.-M. Huang, A. Bhattacharjee, and B. P. Sullivan, Phys. Plasmas 18 072109 (2011).

${ }^{51}$ H. Ji and W. Daughton, Phys. Plasmas 18, 111207 (2011).

${ }^{52}$ M. A. Shay, J. F. Drake, R. E. Denton, and D. Biskamp, J. Geophys. Res. 25, 9176, doi:10.1029/97JA03528 (1998).

${ }^{53}$ B. N. Rogers, R. E. Denton, J. F. Drake, and M. A. Shay, Phys. Rev. Lett. 87, 195004 (2001).

${ }^{54}$ P. A. Cassak, M. A. Shay, and J. F. Drake, Phys. Rev. Lett. 95, 235002 (2005).

${ }^{55}$ L. Zakharov, B. Rogers, and S. Migliuolo, Phys. Fluids B 5, 2498 (1993).

${ }^{56}$ B. Rogers and L. Zakharov, Phys. Plasmas 2, 3420 (1995).

${ }^{57}$ X. Wang, A. Bhattacharjee, and Z. W. Ma, J. Geophys. Res. 105, 27633, doi:10.1029/1999JA000357 (2000).

${ }^{58}$ P. A. Cassak, J. F. Drake, and M. A. Shay, Phys. Plasmas 14, 054502 (2007a).

${ }^{59}$ B. P. Sullivan, A. Bhattacharjee, and Y.-M. Huang, Phys. Plasmas 17, 114507 (2010)

${ }^{60}$ K. Shibata and S. Tanuma, Earth, Planets Space 53, 473 (2001).

${ }^{61}$ W. H. Matthaeus and S. L. Lamkin, Phys. Fluids 28, 303 (1985).

${ }^{62}$ W. H. Matthaeus and S. L. Lamkin, Phys. Fluids 29, 2513 (1986).

${ }^{63}$ A. Lazarian and E. Vishniac, Ap. J. 517, 700 (1999).

${ }^{64}$ Q.-L. Fan, X.-S. Feng, and C.-Q. Xiang, Phys. Plasmas 11, 5605 (2004).

${ }^{65}$ D. Smith, S. Ghosh, P. Dmitruk, and W. H. Matthaeus, Geophys. Res. Lett. 31, L02805, doi:10.1029/2003GL018689 (2004).

${ }^{66}$ G. Kowal, A. Lazarian, E. T. Vishniac, and K. Otmianowska-Mazur, Ap. J. 700, 63 (2009)

${ }^{67}$ N. F. Loureiro, D. A. Uzdensky, A. A. Schekochihin, S. C. Cowley, and T. A. Yousef, Mon. Not. R. Astron. Soc. 399, L146 (2009).

${ }^{68}$ M. Skender and G. Lapenta, Phys. Plasmas 17, 022905 (2010).

${ }^{69}$ M. Hesse, J. Birn, and S. Zenitani, Phys. Plasmas 18, 042104 (2011b).

${ }^{70}$ P. A. Cassak, M. A. Shay, and J. F. Drake, Phys. Plasmas 17, 062105 (2010).

${ }^{71}$ M. A. Shay and J. F. Drake, Geophys. Res. Lett. 25, 3759, doi:10.1029/ 1998GL900036 (1998).

${ }^{72}$ M. Hesse, K. Schindler, J. Birn, and M. Kuznetsova, Phys. Plasmas 6, 1781 (1999).

${ }^{73}$ J. D. Huba and L. I. Rudakov, Phys. Rev. Lett. 93, 175003 (2004). 
${ }^{74}$ M. A. Shay, J. F. Drake, and M. Swisdak, Phys. Rev. Lett. 99, 155002 (2007).

${ }^{75}$ L. S. Shepherd and P. A. Cassak, J. Geophys. Res. 117, A10101, doi:10.1029/2012JA017867 (2012).

${ }^{76}$ P. A. Cassak, J. F. Drake, M. A. Shay, and B. Eckhardt, Phys. Rev. Lett. 98, 215001 (2007b).

${ }^{77}$ L. S. Shepherd and P. A. Cassak, Phys. Rev. Lett. 105, 015004 (2010).

${ }^{78}$ M. Pretko, M. Swisdak, P. A. Cassak, and J. F. Drake, "Investigation of the transition between plasmoid-dominated and Hall magnetic reconnection" (unpublished).

${ }^{79}$ L. Spitzer and R. Härm, Phys. Rev. 89, 977 (1953).

${ }^{80}$ F. L. Waelbroeck, Phys. Fluids B 1, 2372 (1989).

${ }^{81}$ D. W. Longcope and S. C. Cowley, Phys. Plasmas 3, 2885 (1996).

${ }^{82}$ J. K. Edmondson, S. K. Antiochos, C. R. DeVore, and T. H. Zurbuchen, Ap. J. 718, 72 (2010).

${ }^{83}$ M. A. Shay, J. F. Drake, M. Swisdak, and B. N. Rogers, Phys. Plasmas 11, 2199 (2004).

${ }^{84}$ P. A. Cassak, J. F. Drake, and M. A. Shay, Ap. J. 644, L145 (2006).

${ }^{85}$ P. A. Cassak and J. F. Drake, Ap. J. Lett. 707, L158 (2009).

${ }^{86}$ A. N. Simakov, L. Chacón, and D. Knoll, Phys. Plasmas 13, 082103 (2006).
${ }^{87} \mathrm{M}$. Velli, private communication (2010).

${ }^{88}$ A. Ciaravella and J. C. Raymond, Ap. J. 686, 1372 (2008).

${ }^{89}$ J. Lin, J. Li, Y.-K. Ko, and J. C. Raymond, Ap. J. 693, 1666 (2009).

${ }^{90}$ D. E. McKenzie and H. S. Hudson, Ap. J. 519, L93 (1999).

${ }^{91}$ D. E. McKenzie, Sol. Phys. 195, 381 (2000).

${ }^{92}$ L. J. Guo, A. Bhattacharjee, and Y.-M. Huang, in SHINE Workshop oral presentation, 2012.

${ }^{93}$ J. F. Drake, M. Swisdak, K. M. Schoeffler, B. N. Rogers, and S. Kobayashi, Geophys. Res. Lett. 33, L13105, doi:10.1029/2006GL025957 (2006).

${ }^{94}$ W. Daughton, J. Scudder, and H. Karimabadi, Phys. Plasmas 13, 072101 (2006)

${ }^{95}$ R. L. Fermo, J. F. Drake, and M. Swisdak, Phys. Rev. Lett. 108, 255005 (2012)

${ }^{96}$ R. L. Fermo, J. F. Drake, and M. Swisdak, Phys. Plasmas 17, 010702 (2010).

${ }^{97}$ D. A. Uzdensky, N. F. Loureiro, and A. A. Schkochihin, Phys. Rev. Lett. 105, $235002(2010)$

${ }^{98}$ G. L. Delzanno and J. M. Finn, Phys. Plasmas 15, 032904 (2008).

${ }^{99}$ Y.-M. Huang and E. G. Zweibel, Phys. Plasmas 16, 042102 (2009)

${ }^{100}$ D. I. Pontin and Y.-M. Huang, Ap. J. 756, 7 (2012). 